\title{
Scenario-based optimal reserve dispatch considering uncertainty of wind power
}

\author{
L. Y. Chen ${ }^{*}$, Z. Y. Li \\ Department of Electrical Engineering, Zhejiang University, Hangzhou 310027, China
}

\begin{abstract}
This paper presents a model of scenario-based optimal reserve dispatch and security-constrained unit commitment. The objective of the model is to minimize the cost of the energy and reserve. The constraints include network security constraints, reserve scheduled and deployed constraints. The wind speed fluctuation is generated by Monte Caro simulation with Latin hypercube sampling technique. A computationally available mixed-integer linear programming algorithm has been applied to solve the model efficiently. We test our model on the New England 39-bus 10-unit system, and we show the effectiveness of the proposed model for achieving the optimal reserve capacity, economic dispatch and unit commitment.
\end{abstract}

Keywords: Uncertain scenarios, Monte Carlo simulation, scenario reduction, optimal reserve, econimic dispatch; security-constrained unit commitment, mixed-integer linear programming

\section{Introduction}

Due to the wind fluctuation and random failure of thermal units and transmission lines, the independent system operator (ISO) should purchase enough reserve capacity to guarantee the security operation of power system in the day-ahead scheduling planning. The optimal reserve dispatch model has important theoretical significance and practical value [1].

At present, a lot of researches about optimal spinning reserve capacity are carried out. Reference [2] proposes a mathematical model to determine optimal spinning reserve capacity by taking the purchase cost of minimized spinning reserve as objective as well as the secure requirement as chance-constrained. Reference [3] analyzes the optimal accident reserve capacity (in economic sense) in power market environment should be determined in light of cost of accident reserve capacity and load-shedding. A new allocation method is suggested in [4], i.e. the equivalent utilization ratio is formed on the basis of reserve pre-set scheme and the reserve cost is allocated on the basis of the equivalent utilization ratio. Reference [5] presented modeling optimal reserve of interconnected regional power systems based on immune genetic algorithms, placed emphasis on derivative real power increment equation of the tie lines and derivative the connected expression between offset frequency and optimal reserve capacity. The proposed solution for the economic power dispatch and spinning reserve scheduling in [2]-[5] is based on the given unit commitment planning. Reference [6] chooses the bidding output and bidding price to get the maximum profit using the probability theory based on the forecast of the bidding strategy. Reference [7] obtains the income estimate of the power units in energy and reserve markets by using the probability power system production simulation. An effective ac corrective/preventive contingency dispatch over a 24-h period is proposed in [8] based on security-constrained unit commitment model. [9] introduces a security-constrained unit commitment model with emphasis on the simultaneous optimization of energy and ancillary services markets. The optimal reserve dispatch in [8] and [9] is under the premise of the given total reserve capacity.

\footnotetext{
* Manuscript received July 11, 2013; revised August 16, 2013.

Corresponding author. Tel.: 13656698129; E-mail address: clyyuanzi@126.com.
} 
However, research comprehensively studying about optimal reserve dispatch and SCUC considering wind energy is still rare. This paper builds the optimal reserve dispatch model based on security constrained unit commitment (SCUC). The objective of the model is to minimize the cost of the energy and reserve. The constraints include general SCUC constraints, up and down spinning reserve constraints. The wind fluctuation is generated by Monte Caro simulation with Latin hypercube sampling technique. The scenario reduction technique is employed to decrease the computational burden.

As well as unit commitment and economic dispatch, the reserve capacity and dispatch is determined by contingency scenarios. Considering the fault outage of generators, the reserve capacity cannot be allocated on some generators. While considering the failure of transmission lines, the reserve dispatch cannot result in power flow violation on transmission lines. Consequently, the following study is important for building an optimal reserve dispatch model: 1) reserve allocation is combined with power energy dispatch; 2) the effect of network constraints on reserve allocation is considered; 3) the wind fluctuation, described by reduced scenarios, is taken into account.

The formulation of this model is nonlinear. It should be transferred into the mixed integer linear programming (MILP) problem, which is able to be solved by CPLEX12.4 under the circumstance of YALMIP in Matlab10b. The New England 39-bus system with 10 units is analyzed to illustrate the proposed model.

The rest of this paper is organized as follows. Section 2 presents the scenario generation and reduction techniques. Section 3 proposes the formulation of the problem and the solution methodology. Six cases are studied in Section 4. Section 5 concludes the discussion.

\section{Scenario Generation and Reduction}

To capture volatility, we assume the wind speed is subject to a normal distribution $N\left(\mu, \sigma^{2}\right)$ with forecasted wind power as its expected value $(\mu)$ and a percentage of $\mu$ as its volatility $(\sigma)$. Other statistical distributions can be similarly considered. The Monte Carlo simulation will generate a large number of scenarios subject to a normal distribution. The probability of each scenario is one divided by the number of generated scenarios. To decrease the variance of simple Monte Carlo simulation, the Latin hypercube sampling (LHS) technique is employed. The idea in applying LHS is to distribute each sample as the only one in each axis-aligned hyper-plane. LHS can approximate the required normal distribution much better than the simple Monte Carlo method [10].

The scenario reduction technique is employed to decrease the computational requirement for simulating a large number of scenarios. Scenario reduction will eliminate scenarios with very low probability and aggregate close scenarios by measuring the distance between scenarios based on norm operator [11].

\section{Problem Formulation and Solution}

\subsection{Objective}

Assuming both electric energy and reserve ancillary services clear in a single purchasing mode of the electricity market. ISO makes the day-ahead scheduling by minimize the sum cost of energy and reserve capacity. The objective can be written as:

$$
\begin{aligned}
F & =\sum_{t=1}^{n t} \sum_{i=1}^{n g}\left(C_{i, t}^{G}+C_{i, t}^{S U}\right)+\sum_{t=1}^{n t} \sum_{i=1}^{n g}\left(C_{i, t}^{R^{U}} R_{i, t}^{U}+C_{i, t}^{R^{D}} R_{i, t}^{D}\right) \\
& +\sum_{s=1}^{n s} \rho_{s} \sum_{t=1}^{n t}\left(\sum_{i=1}^{n g}\left(C_{i, t, s}^{A}+C_{i, t, s}^{r_{G}}\right)+\sum_{i=1}^{n w}\left(\alpha \Delta w_{i, t, s}\right)+\sum_{i=1}^{n d}\left(\beta \Delta d_{i, t, s}\right)\right)
\end{aligned}
$$

where $C_{i, t}^{G}$ and $C_{i, t}^{S U}$ are the scheduled operation cost and the start-up cost of unit $i$ in period $t(\$)$, respectively; $R_{i, t}^{U}$ and $R_{i, t}^{D}$ are the spinning reserve up and spinning reserve down scheduled for unit $i$ in 
period $t(\mathrm{MW})$, respectively; $C_{i, t}^{R^{U}}, C_{i, t}^{R^{D}}$ are the cost of the up-spinning reserve and down-spinning reserve submitted by unit $i$ in period $t ; \rho_{s}$ is the probability of wind speed scenario s; $C_{i, t, s}^{A}$ is the cost due to change in the operation and start-up plan of unit $i$ in period $t$ and scenario s; $C_{i, t, s}^{r_{G}}$ is the cost of reserve deployed offered by unit $i$ in period $t$ and scenario $s ; \Delta w_{i, t, s}$ is the spillage of wind power; $\Delta d_{i, t, s}$ is the load shedding imposed on consumer $i$ in period $t$ and scenario $s ; \alpha$ and $\beta$ are the value of wind spillage and load shed.

\subsection{Constraints}

The hourly SCUC constraints listed below include the system power balance constraints (2), system spinning reserve requirements and unit power limits (3), spinning reserve limits (4); network security constraints (5), unit minimum ON and OFF time limits (6), unit ramping up and down limits (7), the linearized start-up cost constraints (8). The scenario constraints (9)-(18) represent the system power balance constraints (9), network security constraints (10), unit power limits considering the fault outage of units (11), the real power generation employing the reserve (12), the reserve deployed limits (13), the adjustment of real power generation and start-up cost (14), wind spillage bounds (15); load shedding bounds (16), constraints (17) constitute binary variable declarations.

$$
\begin{aligned}
& \sum_{i=1}^{n g} P_{i, t}+\sum_{i=1}^{n w} W_{i, t}=\sum_{i=1}^{n d} D_{i, t}, \quad \forall t . \\
& U_{i, t} P_{i, \min }+R_{i, t}^{D} \leq P_{i, t} \leq U_{i, t} P_{i, \max }-R_{i, t}^{U}, \quad \forall i, \forall t . \\
& 0 \leq R_{i, t}^{U} \leq\left(P_{i, \max }-P_{i, \min }\right) U_{i, t}, \quad \forall i, \forall t . \\
& 0 \leq R_{i, t}^{D} \leq\left(P_{i, \max }-P_{i, \min }\right) U_{i, t}, \quad \forall i, \forall t . \\
& -\overline{f_{k}} \leq \sum_{n=1}^{n b} T_{i, k}\left(P_{n, t}+W_{n, t}-D_{n, t}\right) \leq \overline{f_{k}}, \quad \forall i, \forall t . \\
& \left(U_{i, t-1}-U_{i, t}\right)\left(t_{\text {on,i,t }}-T_{\text {on,i }}\right) \geq 0, \quad \forall i, \forall t . \\
& \left(U_{i, t}-U_{i, t-1}\right)\left(t_{\text {off }, i, t}-T_{\text {off }, i}\right) \geq 0, \quad \forall i, \forall t . \\
& P_{i, t}-P_{i, t-1} \leq U_{i, t-1}\left(r_{i}^{U}-s_{i}^{U}\right)+s_{i}^{U}, \quad \forall i, \forall t . \\
& P_{i, t-1}-P_{i, t} \leq U_{i, t}\left(r_{i}^{D}-s_{i}^{D}\right)+s_{i}^{D}, \quad \forall i, \forall t . \\
& C_{i, t}^{S U} \geq S_{i, t}\left(U_{i, t}-U_{i, t-1}\right), \quad \forall i, \forall t . \\
& C_{i, t}^{S U} \geq 0, \quad \forall i, \forall t .
\end{aligned}
$$

For scenario simulation:

$$
\begin{aligned}
& \sum_{i=1}^{n g} P_{i, t, s}+\sum_{i=1}^{n w}\left(W_{i, t, s}-\Delta w_{i, t, s}\right)=\sum_{i=1}^{n d}\left(D_{i, t}-\Delta d_{i, t, s}\right), \forall i, \forall t, \forall s . \\
& -\overline{f_{k}} \leq \sum_{n=1}^{n b} T_{i, k, s}\left[P_{n, t, s}+\left(W_{n, t, s}-\Delta w_{n, t, s}\right)-\left(D_{n, t}-\Delta d_{n, t, s}\right)\right] \leq \overline{f_{k}}, \quad \forall i, \forall t . \\
& y_{i, t, s} P_{i, \min } \leq P_{i, t, s} \leq y_{i, t, s} P_{i, \max }, \forall i, \forall t, \forall s . \\
& P_{i, t, s}=P_{i, t}+r_{i, t, s}^{U}-r_{i, t, s}^{D}, \forall t, \forall s, \forall i \in G(t, s) . \\
& 0 \leq r_{i, t, s}^{U} \leq y_{i, t, s} R_{i, t}^{U}, \quad \forall i, \forall t, \forall s . \\
& 0 \leq r_{i, t, s}^{D} \leq y_{i, t, s} R_{i, t}^{D}, \quad \forall i, \forall t, \forall s . \\
& -r_{i}^{D} \leq P_{i, t, s}-P_{i, t-1, s} \leq r_{i}^{U}, \forall i, \forall t, \forall s . \\
& C_{i, t}^{A}=C_{i, t, s}^{G}-C_{i, t}^{G}, \quad \forall i, \forall t, \forall s .
\end{aligned}
$$




$$
\begin{array}{ll}
0 \leq \Delta w_{i, t, s} \leq W_{i, t, s}, & \forall i, \forall t, \forall s . \\
0 \leq \Delta d_{i, t, s} \leq D_{i, t, s}, & \forall i, \forall t, \forall s . \\
U_{i, t}, U_{i, t, s} \in\{0,1\}, & \forall i, \forall t, \forall s .
\end{array}
$$

where $P_{i, t}, D_{i, t}$ are the power of unit $i$ and load of bus $i$ in period $t(M W)$, respectively; $P_{i, \min }, P_{i, \max }$ are the minimum and maximum power of unit $i ; T_{n, k}$ is the power transmission distribution factors of node $n$ in line $k ; y_{i, t, s}$ is the unit forced outage rate; $U_{i, t}$ is $0 / 1$ variable equal to 1 if unit $i$ is scheduled to be committed in period $t ; T_{o n, i}, T_{o f f, i}$ are the minimum on and off time of unit $i ; r_{i}^{U}, r_{i}^{D}, s_{i}^{U}, s_{i}^{D}$ are ramping up and down in continuous operation and start-up operation; $S_{i, t}$ is the start-up cost of unit $i$ in period $t$; $r_{i, t, s}^{U}, r_{i, t, s}^{D}$ are the up-spinning and down-spinning deployed offered by unit $i$ in period $t$ and scenario $s$.

\subsection{Linearization and Solution}

The formulation listed above is nonlinear. It should be transferred into the mixed integer linear programming (MILP) problem, referring to the method proposed in [12]. The analytic representation of the linear approximation of quadratic production cost function is written in (19). The decomposition of the reserve deployment is analogous to production cost, described as (20).

$$
\begin{aligned}
& \Delta P_{G i}=\frac{1}{M}\left(P_{i, \max }-P_{i, \min }\right), \quad \forall i . \\
& 0 \leq p_{i, t}(m) \leq \Delta P_{G i}, \quad \forall m, \forall i, \forall t . \\
& A_{i}=a_{i} P_{i, \min }^{2}+b_{i} P_{i, \min }+c_{i}, \quad \forall i . \\
& P_{i, t}=P_{i, \min } U_{i, t}+\sum_{m=1}^{M} p_{i, t}(m), \quad \forall i, \forall t . \\
& C_{i, t}^{G}=A_{i} U_{i, t}+\sum_{m=1}^{M} k_{m} p_{i, t}(m), \quad \forall i, \forall t . \\
& r_{i, t, s}^{U}-r_{i, t, s}^{D}=\sum_{m=1}^{M} r_{G, i, t, s}(m), \quad \forall i, \forall t, \forall s . \\
& 0 \leq p_{i, t}(m)+r_{G, i, t, s}(m) \leq \Delta P_{G, i}, \quad \forall m, \forall i, \forall t, \forall s . \\
& C_{i, t, s}^{r_{G}}=\sum_{m=1}^{M} k_{m} r_{G, i, t, s}(m), \quad \forall i, \forall t, \forall s .
\end{aligned}
$$

where $M$ is the number of decomposition segment of quadratic production cost through the piecewise linear approximation. $p_{i, t}(m)$ and $r_{G, i, t, s}(m)$ present, respectively, the power produced and the reserve deployed from the $m$ th segment of the energy offered by unit $i$ in period $t$ and scenario $s$.

Finally, (1)-(20) constitute the model of scenario-based optimal reserve dispatch and securityconstrained unit commitment, which is able to be solved by CPLEX12.4 under the circumstance of YALMIP in Matlab10b on a ASUS K45 with two processors at $2.60 \mathrm{GHz}$ and $4 \mathrm{~GB}$ of RAM memory.

\section{Case Studies}

The New England 39-bus 10-unit system in Fig. 1 is used for testing the proposed model that considers the volatility of wind and random failure of generation units and transmission lines. The impact of these uncertainties is analyzed based on economic indices such as operation costs, start-up costs, reserve capacity costs and system security. Different wind volatility is considered to be study the impact on the power system operation. We study six cases as follows:

Case 1): Dispatch without wind generation

Case 2): Dispatch without wind generation uncertainty 
Case 3): Dispatch with uncertain wind generation

Case 4): Dispatch with random failure of thermal units

Case 5): Dispatch with random failure of transmission lines

Case 6): Dispatch with 3), 4) and 5)

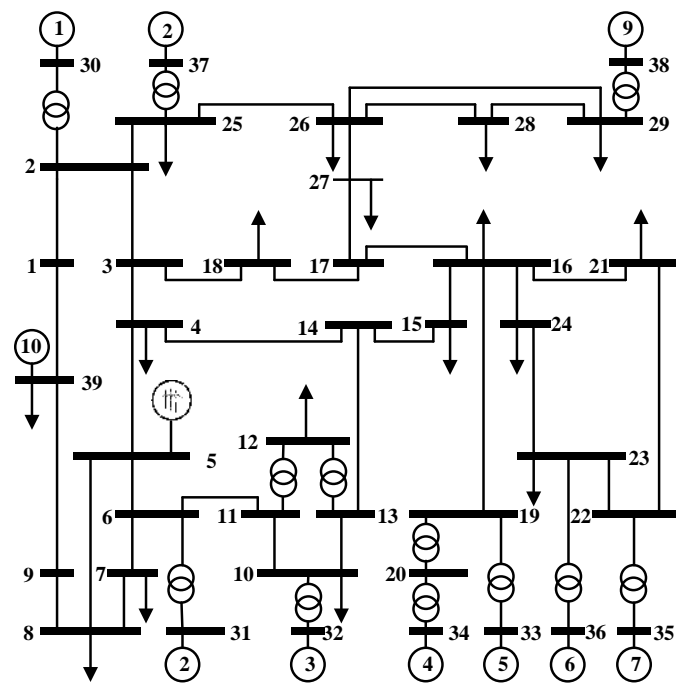

Fig. 1. The New England 39-bus 10-unit system.

Table 1. Reserve dispatch in cases

\begin{tabular}{ccccccc}
\hline \multirow{2}{*}{ Unit } & \multicolumn{7}{c}{ Reserve dispatch results (MW) } \\
\cline { 2 - 7 } & Case 1 & Case 2 & Case 3 & Case 4 & Case 5 & Case 6 \\
\hline 1 & 0 & 0 & 43.2 & 0 & 23.7 & 83.7 \\
2 & 0 & 0 & 55.5 & 33.3 & 28.9 & 0 \\
3 & 0 & 0 & 0 & 0 & 0 & 80.5 \\
4 & 0 & 0 & 56.7 & 33.3 & 14.5 & 0 \\
5 & 0 & 0 & 0 & 0 & 0 & 49.8 \\
6 & 0 & 0 & 26.0 & 16.7 & 21.3 & 0 \\
7 & 0 & 0 & 0 & 0 & 0 & 0 \\
8 & 0 & 0 & 14.7 & 0 & 13.4 & 65 \\
9 & 0 & 0 & 0 & 28.9 & 0 & 14.7 \\
10 & 0 & 0 & 13.3 & 0 & 0 & 40.0 \\
\hline
\end{tabular}

Table 2. Cost in cases

\begin{tabular}{cccc}
\hline Case & Total Cost & Energy Cost & Reserve cost \\
\hline Case 1 & 12549.7 & 12949.7 & 0 \\
Case 2 & 12134.5 & 12534.5 & 0 \\
Case 3 & 13078.2 & 12512.2 & 566 \\
Case 4 & 12956.3 & 12453.3 & 503 \\
Case 5 & 12765.6 & 12287.6 & 478 \\
Case 6 & 13129.3 & 12413.0 & 716.3 \\
\hline
\end{tabular}

\section{Conclusion}

This paper presents a model of scenario-based optimal reserve dispatch and security-constrained unit commitment. The objective of the model is to minimize the cost of the energy and reserve. The constraints include network security constraints, reserve scheduled and deployed constraints. The wind speed fluctuation is generated by Monte Caro simulation with Latin hypercube sampling technique. A computationally available mixed-integer linear programming algorithm has been applied to solve the model efficiently. The case analysis shows the effectiveness of the proposed model for achieving the optimal reserve capacity, economic dispatch and unit commitment. 


\section{References}

[1] Shu J, Li C, Su L, C, Dong W. Optimal reserve dispatch model considering complicated contingency scenarios. Proceedings of the Chinese Society of Electrical Engineering, 2012; 32(10):105-110 (in Chinese).

[2] Wang L, Yu CW, Wen FS. A chance-constrained programming approach to determine requirement of optimal spinning reserve capacity. Power System Technology, 2006; 30(20):14-19 (in Chinese).

[3] Tan LN, Zhang BH. Reserve capacity for accident in power market environment. Proceedings of the Chinese Society of Electrical Engineering, 2002; 22(11):54-58 (in Chinese).

[4] Wang JX, Wang XF, Ding XY. Study on zonal reserve model in power market. Proceedings of the Chinese Society of Electrical Engineering, 2006; 26(18):28-33 (in Chinese).

[5] Wu JK, et al. Immune genetic algorithms for modeling optimal reserve capacity of interconnected regional power systems. Proceedings of the Chinese Society of Electrical Engineering, 2009; 29(1):14-20 (in Chinese).

[6] Ren Z., Wu G, Huang W. The research of the bidding strategy of generation considering the spinning reserve. Proceedings of the Chinese Society of Electrical Engineering, 2004. 24(12):48-51 (in Chinese).

[7] Zou B, et al. Unbunding condition of pricing methods between energy market and reserve market. Proceedings of the Chinese Society of Electrical Engineering, 2003. 23(12): (in Chinese).

[8] Fu Y, Shahidehpour M, Li Z. AC contingency dispatch based on security-constrained unit commitment. IEEE Transactions on Power Systems, 2006; 21(2):897-908.

[9] Li Z. Shahidehpour M. Security-constrained unit commitment for simultaneous clearing of energy and ancillary services markets. IEEE Transactions on Power Systems, 2005; 20(2):1079-1088.

[10] Wang J, Shahidehpour M, Li Z. Security-constrained unit commitment with volatile wind power generation. IEEE Transactions on Power Systems, 2008; 23(3):1319-1327.

[11] Gröwe-Kuska N, Heitsch H, Romisch W. Scenario reduction and scenario tree construction for power management problems. Presented at: IEEE Power Tech Conf., 2003.

[12] Carrion M, et al. A computationally efficient mixed-integer linear formulation for the thermal unit commitment problem. IEEE Transactions on Power Systems, 2006; 21(3):1371-1378. 